\title{
Análisis comparativo de la función del injerto renal de donante vivo tras nefrectomía abierta y laparoscópica: modelo experimental
}

\author{
Linares Quevedo AI*, Burgos Revilla FJ**, Villafruela Sanz JJ, Zamora Romero J****, \\ Pascual Santos $\mathrm{J}^{* * *}$, Marcén Letosa $\mathrm{R}^{* * *}$, Cuevas Sánchez B****, Correa Gorospe $\mathrm{C}^{\star * * *}$. \\ *Servicio de Urología. Hospital de Fuenlabrada. ${ }^{* *}$ Servicios de Urología, ${ }^{* * *}$ Nefrología y ${ }^{* * * * U n i d a d ~ d e ~ A p o y o ~ a ~}$ \\ la Investigación. Hospital Ramón y Cajal. Universidad de Alcalá. Madrid.
}

Actas Urol Esp. 2008;32(1):140-151

\section{RESUMEN}

ANÁLISIS COMPARATIVO DE LA FUNCIÓN DEL INJERTO RENAL DE DONANTE VIVO TRAS NEFRECTOMÍA ABIERTA Y LAPAROSCÓPICA: MODELO EXPERIMENTAL

Introducción: El trasplante renal (TR) de donante vivo presenta una supervivencia del injerto y del paciente superior al TR procedente de cadáver y representa una alternativa eficaz frente al problema de la escasez de órganos.

Objetivo: Analizar comparativamente en un modelo experimental en cerdo, la influencia del síndrome de isquemia repercusión y la evolución funcional del injerto renal extraído mediante nefrectomía abierta y laparoscópica.

Material y métodos: 30 cerdos fueron sometidos a nefrectomía izquierda: 15 por laparoscopia y 15 por vía abierta, como donantes vivos en un modelo de autotrasplante renal. Se midió el flujo sanguíneo renal (FSR) postdesclampaje mediante sonda electromagnética y los niveles de creatinina $(\mathrm{Cr})$ durante la primera semana postrasplante.

Resultados: El análisis comparativo del FSR en la $1^{\text {a }}$ hora postTR objetivó: una disminución significativa del FSR medio en el grupo de laparoscopia frente al abierto $(\mathrm{p}<0,001)$, con una reducción del FSR en los primeros 5 minutos más acusado en el grupo laparoscópico $(\mathrm{p}<0,001)$, y una recuperación progresiva del FSR durante la $1^{\mathrm{a}}$ hora, que es más lenta para el grupo laparoscópico. Los niveles de $\mathrm{Cr}$ en la primera semana postrasplante descendieron progresivamente desde 1,3 a $0,8 \mathrm{mg} / \mathrm{dl}$ en el grupo abierto, y de 2 a $1,1 \mathrm{mg} / \mathrm{dl}$ en el grupo laparoscópico $(\mathrm{p}<0,001)$.

Conclusiones: Los injertos renales extraídos vía laparoscópica presentan una acentuación del síndrome de isquemiareperfusión manifestado por un FSR postdesclampaje inferior al FSR prenefrectomía y un deterioro significativo de la función renal durante la primera semana postrasplante.

Palabras clave: Trasplante renal. Nefrectomía abierta. Nefrectomía laparoscópica.

\section{ABSTRACT \\ COMPARATIVE ANALYSIS OF RENAL GRAFT FUNCTION AFTER OPEN VS LAPAROSCOPIC NEPHRECTOMY: EXPERIMENTAL MODEL}

Introduction: Living donor renal transplant reports a higher patient and graft survival in comparison to cadaver donor and represents a good alternative facing the current lack of organs for transplant.

Goals: To analyze comparatively in an experimental model (pig) the influence of ischemia-reperfusion and functional outcome of renal graft retrieved by open Vs laparoscopic nephrectomy.

Material and methods: 30 lab pigs were nephrectomized (left kidney): 15 by laparoscopy and 15 by open surgery, as living donors, in a model of renal autotransplant. Renal blood flow (RBF) was measured by means of an electromagnetic probe and creatinine levels during the first week after the implant.

Results: Comparative analysis of RBF during the immediate $60 \mathrm{~min}$ after unclamping showed a significant reduction of average RBF in laparoscopic group in comparison to open group $(p<0.001)$, with a more evident reduction of $R B F$ in the laparoscopic group during the 5-min period after unclamping $(\mathrm{p}<0.001)$ and a progressive recuperation of RBF during the $1^{\text {st }}$ hour, slowest in laparoscopic group. Creatinine levels in the first week after the transplant decreased progressively from 1.3 to $0.8 \mathrm{mgrs} / \mathrm{dl}$ in the open group and from 2 to $1.1 \mathrm{mg} / \mathrm{dl}$ in laparoscopic group $(\mathrm{p}<0.001)$.

Conclusions: Renal grafts retrieved by laparoscopy presents a more evident ischemia-reperfusion syndrome shown by a lower average RBF after unclamping and a significant deterioration of renal function during the first week after transplant.

Keywords: Renal transplantation. Open nefrectomy. Laparoscopic nefrectomy. 
$\mathrm{E}$ 1 trasplante renal (TR) es el único tratamiento que permite evitar la diálisis en los pacientes con insuficiencia renal terminal. Los pacientes trasplantados tienen una mejor calidad de vida y una mayor supervivencia que los pacientes que permanecen en lista de espera ${ }^{1-3}$. Debido a la escasez de órganos, no es posible llevarlo a cabo en todos ellos: existe una divergencia cada vez mayor entre el número de personas que necesitan un trasplante y el número de las que efectivamente lo reciben.

El TR de donante vivo representa una alternativa eficaz para hacer frente a este problema, pero la resistencia a la donación puede deberse en parte a las inquietudes relacionadas con la cirugía, el tiempo de hospitalización, el periodo prolongado de recuperación, la morbilidad y la estética.

\section{NEFRECTOMÍA DE DONANTE VIVO}

El trasplante de un riñón procedente de un donante vivo obtiene mejores resultados que el trasplante renal de riñón procedente de cadáver, tanto lo que se refiere a la supervivencia del injerto como a la supervivencia del paciente ${ }^{4}$. Pero el empleo de un injerto procedente de donante vivo, tiene dos limitaciones fundamentales:

- Por una lado, el gran crecimiento de la donación de cadáver en España, ha frenado la de vivo. Así, el aumento de la edad de los donantes hace que los injertos marginales representen en la actualidad $30 \%$ del total. Si bien es cierto, que algunos de los órganos extraídos finalmente no son implantados, de hecho, sólo el 73\% de los riñones extraídos son finalmente trasplantados. La tendencia a implantar órganos de donantes añosos en receptores de edad avanzada conlleva que el tiempo de permanencia en lista de espera de pacientes jóvenes en diálisis sea elevado. Para estos pacientes deberian potenciarse otras vías de obtención de órganos, como el donante vivo ${ }^{5}$, sin olvidar que la nefrectomía de donante vivo, no es una cirugía exenta de morbimortalidad en un paciente sano, que no obtiene beneficio terapéutico personal.

- Por otro lado, el TR de donante vivo presenta condicionantes sociales, culturales, económicos, médicos, legales, éticos y religiosos que justifican la diferente incidencia del mismo en los distintos países. En países como Méjico y Turquía, con un porcentaje muy bajo de donante cadáver, el TR de donante vivo representa el 85\% de todos los TR realizados ${ }^{6,7}$.

Actualmente en España, el trasplante de donante vivo se sitúa en torno al $3 \%$ del total de TR, frente al $12 \%$ de la media europea ${ }^{8}$. Muy inferior al 29\% Australiano, al 52\% norteamericano, y por supuesto, al $27 \%$ de Suecia o al $40 \%$ que ha llegado a alcanzar Noruega ${ }^{9,10}$. La tendencia en nuestro país, aunque muy por debajo de la media europea y norteamericana, muestra un lento incremento: de 17 trasplantes de donante vivo en $1999(0,4 \%)$, a 34 en el $2002(0,8 \%)$ y $84(2,8 \%)$ durante el año $2005^{9}$.

La nefrectomía de donante vivo es una técnica que tiene ya medio siglo: en 1952, Vaysse y Oeconomos, llevan a cabo en Paris el primer trasplante de donante vivo emparentado. Pero no es hasta 1954 cuando Murray et al, en el Peter Ben Brighan de Boston, consigue una supervivencia larga al realizar un trasplante entre gemelos univitelinos.

Esta técnica está resurgiendo en todo el mundo, como consecuencia de la posibilidad de emplear donantes no emparentados, gracias a la eficacia de los nuevos inmunosupresores, y a la adaptación de los requisitos legales como ocurrió en España en el año 2000.

Las nuevas técnicas de Nefrectomía laparoscópica de donante vivo (NLDV) pueden, sin duda, contribuir a disminuir la morbilidad derivada del procedimiento y quizás a favorecer su generalización.

La nefrectomía del donante vivo tiene un carácter singular, porque se realiza en un individuo sano, que no obtiene beneficio directo del procedimiento, y su empleo tiene una serie de implicaciones tanto para el receptor como para el donante:

\section{Implicaciones para el receptor}

Los injertos procedentes de donante vivo, presentan numerosas ventajas frente a los de donante cadáver:

\section{Mejor supervivencia del injerto}

Los injertos procedentes de donante vivo, incluso en el caso de los donantes no relacionados, presentan mejor supervivencia a largo plazo que los de donante cadáver, acercándose la misma a la de 
los donantes vivos relacionados que comparten HLA-idénticos o haploidénticos ${ }^{4,11,12}$.

La supervivencia de los injertos procedentes de donante vivo a los 1 y 3 años, son del 93\% y $84 \%$ respectivamente ${ }^{13}$. Estas supervivencias son superiores a las de los injertos de donante cadáver, que se sitúan entre el 88 y $69 \%$ a los mismos periodos de tiempo. La menor incidencia de rechazo agudo y de necrosis tubular aguda (NTA) en el postTR inmediato condiciona la mejor supervivencia del injerto.

La menor incidencia de rechazo viene determinada por dos factores: el mayor grado de identidad entre los antígenos del donante y del receptor, y por otro lado, la menor incidencia de $\mathrm{NTA}^{12}$.

\section{Reducción de inmunosupresión}

La menor incidencia de rechazo, permite disminuir la dosis de medicación inmunosupresora y los efectos secundarios de la misma, como el desarrollo de tumores a largo plazo, o las consecuencias del uso crónico de esteroides ${ }^{12}$.

\section{Menor tiempo en diálisis}

El TR de donante vivo es especialmente importante en la infancia, ya que reduce el tiempo en diálisis, en un momento en el que el crecimiento del niño exige una función renal normal. Por otro lado, permite elegir el momento óptimo para el trasplante en el receptor.

\section{Menor incidencia de NTA}

La reducción del tiempo de isquemia fría a algunos minutos, reduce la necesidad de diálisis en el postTR inmediato a menos del $1 \%^{12,13}$.

\section{Menor coste económico}

En un estudio reciente llevado a cabo por Mullins et $\mathrm{al}^{14}$, comparan el coste del TR de donante vivo mediante nefrectomía laparoscópica, frente al trasplante de cadáver y el tratamiento con diálisis. El coste es claramente inferior para el primer grupo, seguido por el TR de cadáver y finalmente por la diálisis.

\section{Implicaciones para el donante}

La nefrectomía del donante vivo, es segura tanto en función renal a largo plazo como en esperanza de vida ${ }^{15,16}$ fruto de una exhaustiva evaluación para la selección de los donantes. El riesgo de mortalidad en la literatura oscila del 0,06 al $0,03 \%{ }^{17,18}$.

Existe controversia, sobre los efectos secundarios potenciales a largo plazo que la nefrectomía tiene en el donante como el desarrollo de hipertensión arterial (HTA) y el deterioro de la función renal.

Tras la nefrectomía, tiene lugar un descenso moderado del aclaramiento de creatinina $(\mathrm{Cr})$ (desde $109+26 \mathrm{ml} \mathrm{min} \mathrm{a} 78+23 \mathrm{ml} / \mathrm{min}$ ), que mejora gradualmente en función de la edad del paciente, del periodo de seguimiento por hiperfiltración y del desarrollo de hipertrofia compensadora del riñón contralateral ${ }^{17}$.

La presencia de HTA ha sido referida a largo plazo en pacientes uninefrectomizados, especialmente en grupos étnicos de alto riesgo para el desarrollo de la misma. La incidencia de HTA post-nefrectomía, que exige tratamiento médico, oscila en la literatura del 8,8-60\% 95 $5^{16,17}$. Sin embargo, el 8,8\% alcanzado en algunas series es menor que la incidencia de hipertensión en la población general con la misma edad. ${ }^{16}$

\section{Técnicas de nefrectomía en el donante vivo}

La nefrectomía de donante vivo es una intervención, en la que se somete a un sujeto sano, a la morbilidad y mortalidad que conlleva la cirugía invasiva, con el fin de mejorar la calidad de vida y la supervivencia de otra persona, aquejada de insuficiencia renal terminal.

Por ello, son de especial trascendencia la seguridad y la eficacia de la nefrectomía en el donante: la intervención para obtener el riñón debe reducir en todo lo posible la morbilidad inherente a la cirugía, y permitir una reincorporación rápida a la actividad normal.

Para el receptor, la intervención debe proporcionar una unidad renal indemne, sometida al menor tiempo posible a isquemia caliente, y con una longitud suficiente de arteria y vena, sin lesión endotelial. Todo ello posibilitará una función inmediata del injerto, con una baja incidencia de complicaciones vasculares y ureterales.

\section{Nefrectomía abierta}

A lo largo de los últimos 35 años, la nefrectomía de donante vivo a través de una incisión 
abierta ha sido la técnica habitual. La evaluación de una nueva técnica quirúrgica de nefrectomía de donante vivo, exige que los resultados obtenidos sean comparables a los de la cirugía abierta que constituye el patrón de referencia.

El tiempo quirúrgico medio referido es de 2 horas 43 minutos $^{19}$.

La incidencia de complicaciones postquirúrgicas, oscila del el 3,4-7,8\% al $17 \%$ según las series $^{19-21}$.

Najarian et $\mathrm{al}^{18}$, documentan una mortalidad del $0,03 \%$, semejante al 0,06\% referido por Bay et $\mathrm{al}^{17}$.

\section{Nefrectomía laparoscópica transperitoneal}

El desarrollo reciente de la cirugía mínimamente invasiva, potenció la expansión de la nefrectomía laparoscópica de donante vivo (NLDV).

La primera nefrectomía por vía laparoscópica fue descrita por Clayman et al, en $1991^{22}$.

La nefrectomía laparoscópica para TR, fue descrita por primera vez a nivel experimental en un modelo porcino por Gill et al, en $1994^{23}$.

Desde la realización del primer caso de NLDV por Ratner et al. ${ }^{24}$, en el John Hopkins Medical Center de Baltimore en 1995 con injerto funcionante, tras 5 minutos de isquemia caliente, la técnica ha tenido una rápida difusión y en la actualidad, está sustituyendo a la nefrectomía abierta como patrón de referencia para la nefrectomía del donante vivo ${ }^{3}$.

La primera nefrectomía de donante vivo realizada por un grupo exclusivamente urológico, fue en febrero de 2002 en la Fundación Puigvert de Barcelona, alcanzando la cifra de 60 casos en febrero del 2005, publicados recientemente ${ }^{25}$.

LA NLDV reduce significativamente el dolor postoperatorio, la estancia hospitalaria y el tiempo de vuelta al trabajo, con la misma seguridad y eficacia, frente a la cirugía abierta ${ }^{26-29}$.

Sin embargo, los tiempos de quirófano y de isquemia caliente de la NLDV son netamente más $\operatorname{largos}^{11,12,20}$. El tiempo de isquemia caliente medio oscila entre 2 y 7 minutos, pero ello no conlleva un deterioro de la función inicial del injerto ni de los resultados de supervivencia ${ }^{26,27,30,31}$.

La influencia en la función inmediata del injerto de la nefrectomía laparoscópica frente a la cirugía abierta está sometida a controversia.
Comparado con la cirugía abierta, los niveles de $\mathrm{Cr}$ se han visto elevados los primeros 3-6 dias postTR ${ }^{32}$, no obstante, a los 3, 6 y 12 meses no existía diferencia significativa entre los dos grupos. Incluso, tras el año del trasplante, se han descrito niveles inferiores de $\mathrm{Cr}$ en el grupo de nefrectomía laparoscópica frente al de nefrectomía abierta ${ }^{27,32-35}$. Las complicaciones quirúrgicas para el receptor, y la supervivencia del injerto a largo plazo, es similar en ambos grupos ${ }^{36}$.

La primera NLDV mano asistida, empleando el dispositivo Pneumosleeve (Dexterity, Blue Bell, PA,), fue descrita por Nakada et al. ${ }^{37}$ en 1997. La ayuda manual permite reducir notablemente la duración de la intervención ${ }^{38}$, acorta la curva de aprendizaje de la NLDV ${ }^{39,40}$ y reduce las complicaciones intraoperatorias. Respecto al periodo de isquemia caliente, algunos grupos consiguen una isquemia caliente y tiempo quirúrgico equiparable a la de las técnicas abierta ${ }^{41}$. El abordaje mano-asistido, reduce el tiempo quirúrgico y el tiempo de isquemia caliente en la mayoría de los trabajos $^{42,43}$. No se han visto diferencias, en términos de dias de estancia y periodo de recuperación, respecto al abordaje laparoscópico no mano-asistido 44,45 .

Las ventajas para el donante descritas con la NLDV, como publican Ratner et al. ${ }^{46}$, han incrementado hasta un $25 \%$ el número de donantes en Estados Unidos. Un sistema de información y educación dirigido a los potenciales donantes, como describen Schweitzer et al. ${ }^{47}$, aumentaría de forma importante el número de trasplantes de donante vivo. La escasa proporción actual de TR de donante vivo en España, supone un obstáculo para la introducción de la cirugía laparoscópica en este campo, pero la tasa de donación en España es la más alta del mundo (34,6 p.m.p.), y la tasa de negativas familiares es la más baja $(17,8 \%)$, cifras ya dificilmente superables, y por tanto se hace necesario explorar otras vías de obtención de riñones para TR.

\section{Nefrectomia por retroperitoneoscopia}

La primera nefrectomía retroperitoneoscópica, fue realizada por Gaur en $1993^{48}$. Yang et $\mathrm{al}^{49}$, publican el primer caso de nefrectomía retroperitoneal de donante vivo video-asistida. Como se refleja en los estudios de Guillonneau et $\mathrm{al}^{50}$, la 
morbilidad, complicaciones postoperatorias y estancia hospitalaria, eran similares para la NLDV trans o retroperitoneal.

En un intento de combinar las ventajas de la laparoscopia manoasistida, junto con el abordaje retroperitoneal se han publicado buenos resultados con la nefrectomía por retroperitoneoscopia mano-asistida: se han descrito tiempos de isquemia caliente similares a los de la cirugía abierta, y menores a los del abordaje laparoscópico retroperitoneal, con las mismas ventajas ${ }^{51-53}$.

De todo lo dicho anteriormente podemos concretar los siguientes puntos:

1. La modificación del perfil del donante renal, el aumento del número de pacientes en lista de espera para trasplante y las cifras alcanzadas de injerto procedente de donante cadáver, exigen el desarrollo del trasplante procedente de donante vivo.

2. La posibilidad de obtener un riñón de un donante vivo mediante nefrectomía laparoscópica es una realidad. Pero es necesario conocer los efectos que el neumoperitoneo y el periodo de isquemia caliente ejercen sobre el riñón hasta su implante y reperfusión:

- Durante la NLDV existen modificaciones hemodinámicas y una reducción del FSR que puede condicionar la función del injerto en el postrasplante inmediato.

- Presumiblemente, este efecto vendría mediado, entre otros, por acentuación del síndrome de isquemia-reperfusión que conlleva inevitablemente el proceso de trasplante ${ }^{54}$.

El interés práctico de este estudio consiste en conocer la evolución funcional del injerto renal extraído mediante nefrectomía abierta y laparoscópica, así como el síndrome de isquemia reperfusión a través del análisis comparativo del FSR postdesclampaje.

El estudio se realizó mediante un modelo experimental y no clínico, para tener un control adecuado de todas las variables que intervienen, y se escogió un animal de experimentación grande, como el cerdo, de gran similitud con el humano.

\section{MATERIAL Y MÉTODOS}

Los animales de estudio fueron 30 cerdos de raza ibérica, cuyo peso medio fue de $22,6+3,2 \mathrm{~kg}$ (Rango 19-31 kg).
Se estudiaron dos grupos de 15 cerdos cada uno:

Grupo Abierto ( $\mathrm{n}=15$ cerdos). Utilizado como "Grupo Control" en el que el abordaje renal se realizó por cirugía abierta convencional.

Grupo Lap ( $\mathrm{n}=15$ cerdos). Utilizado como "Grupo Estudio" en el que el abordaje se practicó por vía laparoscópica.

En una primera fase se llevó a cabo extracción del injerto renal izquierdo en el donante mediante abordaje abierto o bien laparoscópico, a continuación la nefrectomía contralateral abierta en ambos grupos y a continuación el autotrasplante ortotópico del injerto renal extraído previamente.

El estudio experimental realizado se basa en el siguiente diseño:

1. Premedicación y anestesia general del animal. Monitorización en ambos grupos de la TA y PVC. Se realiza en todos ellos una premedicación con Ketamina intramuscular (10 mg/kg) e inducción anestésica con Tiopental sódico intravenoso $(10 \mathrm{mg} / \mathrm{kg})$ y Atropina $(0,01 \mathrm{mg} / \mathrm{kg})$. Se procede a la intubación endotraqueal y ventilación controlada del animal. La anestesia se mantiene con Halotano al 0,9-1,25\% y $\mathrm{N}_{2} \mathrm{O}$ mezclado con $\mathrm{O}_{2}$. La analgesia intraoperatoria se realiza con Fentanilo a dosis de $50 \mu \mathrm{g} /$ hora.

Se administra de forma preoperatoria Cefazolina 1 gramo intravenoso en dosis única. La hidratación del animal durante la cirugía se realiza mediante la administración de suero glucosalino a dosis de $10 \mathrm{ml} / \mathrm{Kg} /$ hora.

\section{Abordaje Quirúrgico}

2.1. Nefrectomía izda. En el Grupo Abierto: incisión de lumbotomía izquierda bajo la décimosegunda costilla en los 15 animales. En el grupo LAP se realiza abordaje laparoscópico transperitoneal e insuflación con $\mathrm{CO}_{2}$ para mantener una PIA constante de $15 \mathrm{mmHg}$.

2.2. Cirugía de banco. En los 30 riñones izquierdos extraídos previamente, 15 mediante nefrectomía abierta y 15 mediante nefrectomía laparoscópica, se realiza la revisión de la arteria y vena renal izquierdas, para su posterior anastomosis término-terminal en el autotrasplante, así como exérexis de los ganglios linfáticos hiliares. 
Se realiza la perfusión fría de los riñones a través de cánula y sistema de suero con solución de Eurocollins. Ya preparados, se colocan en una bolsa de órganos estéril con solución Eurocollins a $4^{\circ} \mathrm{C}$, y se introducen en una nevera portátil con abundante hielo rodeándola, donde permanecerán en isquemia fría durante 24 horas hasta su implantación en el receptor.

2.3. Nefrectomía abierta derecha y autotrasplante ortotópico. El autotrasplante se lleva a cabo a las 24 horas de isquemia fría en el grupo Abierto y Lap.

Se practica una pequeña incisión de lumbotomía derecha extraperitoneal, realizando la nefrectomía derecha, y se coloca el riñón izquierdo donante en la fosa renal derecha del mismo animal, realizando la anastomosis término-terminal con sutura contínua $180^{\circ}$ con monofilamento irreabsorvible Prolene $7 / 0$ de la vena y arteria renales.

La reconstrucción del tracto urinario, se realiza con sutura continua de Vicryl rapid 4/0 mediante urétero-ureterostomía término-terminal proximal, previa tutorización con catéter doble $\mathrm{J}$ 6/24.

El primer tiempo de isquemia caliente fue de 1,8 minutos en el grupo ABIERTO y de 6,8 minutos en el grupo LAP, el tiempo de isquemia fría fue de 24 horas en ambos grupos, y el segundo tiempo de isquemia caliente fue de 33 y 31 minutos para el grupo ABIERTO y LAP respectivamente.

El sacrificio del animal se realiza al $7^{\circ}$ día del autotrasplante con cloruro potásico $(20 \mathrm{mEq})$ por vía intravenosa.

4. Medidas del FSR postdesclampaje. Tras finalizar las anastomosis vasculares y habiendo desclampado los vasos, se toman las medidas del FSR con sonda electromagnética a los minutos 1 , 5, 10, 20, 30, y 60 tras desclampaje. (Fig. 1).

5. Determinaciones analíticas. En todos los animales, determinaciones de sangre al inicio y posteriormente tras finalizar el autotrasplante ortotópico, mediante venopunción periférica de la vena auricular, en la cara dorsal de la oreja, bajo sedación, los días 1, 3, 5 y 7 del autotrasplante.

Para determinar los valores de $\mathrm{Cr}$, se obtuvieron en cada momento $6 \mathrm{cc}$ de sangre que se depositaron en un tubo vacío (Vacutainer). Se centri-

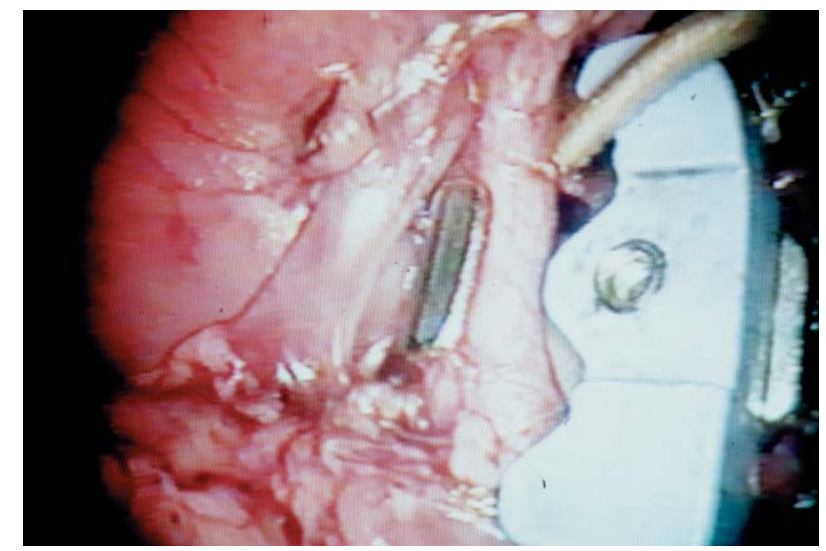

FIGURA 1. Medida del FSR en la arteria renal izquierda.

fugaron a $4.000 \mathrm{rpm}$ durante 10 minutos; el plasma se almacenó en alícuotas de $1 \mathrm{ml}$ en microtubos Eppendorf y se congeló a $-20^{\circ}$.

6. Análisis estadístico de los resultados. Como estimador de la tendencia central de las variables se utilizó la media, y como estimador de la dispersión se empleó la desviación estándar. Para comparar la diferencia entre las medias, se utilizó el test de la t de Student.

Para todas las comparaciones se empleó un nivel de significación estadística del 95\%.

\section{RESULTADOS}

Sindrome de isquemia-reperfusión. Análisis comparativo del FSR postdesclampaje entre nefrectomía abierta y laparoscópica

La influencia de la nefrectomía laparoscópica en el sindrome de isquemia reperfusión, se analizó en base al análisis comparativo del FSR tras el desclampaje.

La Figura 2 y la Tabla 1 muestran la evolución del FSR tras la revascularización del injerto renal al finalizar el autotrasplante de modo comparativo entre la cirugia abierta y laparoscópica.

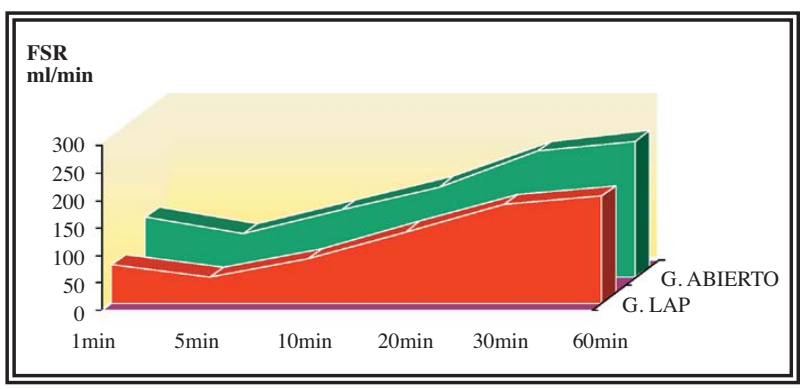

FIGURA 2. FSR postdesclampaje en injertos extraidos mediante nefrectomia abierta y laparoscópica. 
Tabla 1. Valores de FSR postdesclampaje de los injertos renales obtenidos por vía abierta y laparoscópica.

\begin{tabular}{lcccc}
\hline TIEMPO (min) & & $\begin{array}{c}\text { G. ABIERTO } \\
\mathrm{n}=15\end{array}$ & $\begin{array}{c}\text { G. LAP } \\
\mathrm{n}=15\end{array}$ & $\mathbf{p}$ \\
\hline FSR & 1 & $111 \pm 1,7$ & $70 \pm 1,7$ & $<0,001$ \\
Postdesclampaje & 5 & $80 \pm 1,6$ & $46 \pm 1,3$ & $<0,001$ \\
$(\mathrm{ml} / \mathrm{min})$ & 10 & $123 \pm 1,9$ & $80 \pm 1,6$ & $<0,001$ \\
& 20 & $165 \pm 2,1$ & $130 \pm 1,4$ & $<0,001$ \\
& 30 & $230 \pm 2,5$ & $180 \pm 1,9$ & $<0,001$ \\
& 60 & $249 \pm 2,5$ & $195 \pm 1,8$ & $<0,001$ \\
\hline & FSR & $159,7 \pm 6,6$ & $117,0 \pm 5,7$ & $<0,001$ \\
& medio & & & \\
\hline
\end{tabular}

El análisis comparativo del FSR en la $1^{\text {a }}$ hora postTR objetivó:

- Una disminución significativa del FSR medio durante la primera hora en el grupo de laparoscopia frente al de cirugía abierta $(\mathrm{p}<0,001)$.

- Una reducción del FSR en los primeros 5 minutos que es más acusado en el grupo con nefrectomía laparoscópica que con nefrectomía abierta $(46+1,3 \mathrm{ml} / \mathrm{min}$ vs $80+1,6 \mathrm{ml} / \mathrm{min}$ a los 5 minutos; $\mathrm{p}<0.001)$.

- Una recuperación progresiva del FSR durante la $1^{a}$ hora, que es más lenta para el grupo LAP.

\section{Evolución funcional del injerto. Análisis comparativo entre nefrectomia abierta y laparoscópica}

Los niveles de $\mathrm{Cr}$ medidos los días 1, 3, 5, y 7 postrasplante descendieron progresivamente desde 1,3 a 0,8 mg/dl en el grupo ABIERTO, y de 2 a $1,1 \mathrm{mg} / \mathrm{dl}$ en el grupo LAP. La diferencia entre ambos grupos fue significativa $(\mathrm{p}<0,001)$.

Los valores de $\mathrm{Cr}$ plasmática tras el autotrasplante, los días 1, 3, 5, y 7 en ambos grupos se muestran en la Figura 3 y en la Tabla 2.

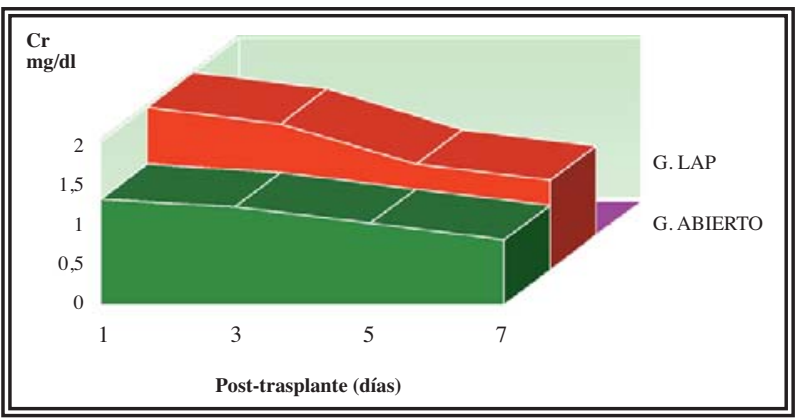

FIGURA 3. Análisis comparativo de los valores de $\mathrm{Cr}$ en el postrasplante de los injertos procedentes de nefrectomía laparoscópica y nefrectomía abierta.

\section{DISCUSIÓN}

Existen estudios retrospectivos comparando la NLDV frente a la nefrectomía abierta, que demuestran una función similar del injerto en ambos grupos al año del trasplante, pero con unos niveles más elevados de creatinina y de diálisis en el grupo de nefrectomia laparoscópica en las primeras semanas postTR $2,55,56$.

Distintos mecanismos han sido implicados en la disfunción inicial de los injertos extraídos por vía laparoscópica, entre ellos el mayor tiempo de isquemia caliente y los efectos que el neumoperitoneo ejerce sobre el riñón ${ }^{56,57}$. Diversos estudios, tanto en modelos experimentales de animal grande $54,58,59$ como pequeño ${ }^{60}$ han revelado una disminución del FSR con el aumento de la PIA; lo que favorecería la oliguria y la NTA ${ }^{61}$. En nuestro modelo experimental, durante la nefrectomía izquierda laparoscópica los animales experimentaron significativamente una disminución del $69 \%$ del FSR, con una disminución significativa de la diuresis respecto a la basal superior al 40\%, y un descenso significativo del filtrado glomerular (FG) del 38\%, a los 30 y 60 minutos de neumoperitoneo respecto al grupo abierto ${ }^{62}$.

Sin embargo, los efectos del neumoperitoneo sobre la función renal de los injertos renales extraídos por vía laparoscópica no ha sido aún completamente estudiada, estando algunos aspectos sometidos a controversia.

En nuestro modelo, se realizó la nefrectomía laparoscópica del donante vivo, y posteriormente autotrasplante ortotópico. El impacto del neumoperitoneo en el sindrome de isquemia reperfusión y en la evolución funcional del injerto extraído

Tabla 2. Valores de $\mathrm{Cr}$ en ambos grupos durante la semana posterior al autotrasplante

\begin{tabular}{lcccc}
\hline & $\begin{array}{c}\text { Postrasplante } \\
\text { (dias) }\end{array}$ & $\begin{array}{c}\text { G. Abierto } \\
\mathrm{n}=15\end{array}$ & $\begin{array}{c}\text { G. LAP } \\
\mathrm{n}=15\end{array}$ & $\mathbf{p}$ \\
\hline $\mathbf{C r}$ & Basal & $0,8+0,4$ & $0,7+0,0$ & $<0,001$ \\
$\mathbf{m g} / \mathbf{d} \mathbf{1}$ & $\mathbf{1}$ & $1,3+0,1$ & $2,0+0,1$ & $<0,001$ \\
& $\mathbf{3}$ & $1,2+0,1$ & $1,8+0,1$ & $<0,001$ \\
& $\mathbf{5}$ & $1,0+0,1$ & $1,3+0,1$ & $<0,001$ \\
\hline & $\mathbf{7}$ & $0,8 \pm 0,1$ & $1,1 \pm 0,1$ & $<0,001$ \\
\hline
\end{tabular}


por vía laparoscópica, apenas se ha realizado en el modelo experimental animal, sino que en la literatura se refleja en las series de casos clínicos de los principales centros donde se realiza la NLDV $10,19,21,23,26,30,32,35,36$. En definitiva, se ha intentado reproducir, con la máxima similitud posible y reduciendo al máximo el número de variables, aquella situación clínica que se daría en el humano. Como único factor criticable, que no se reproduce exactamente en el TR con donante vivo en el humano, se encuentra el someter el injerto a un periodo de isquemia fría de 24 horas. Esta circunstancia fue una exigencia del adiestramiento laparoscópico inicial del grupo, de la puesta a punto del modelo experimental y de la exigencia de estudiar las modificaciones inducidas por distintos ciclos de insuflación y desinsuflación. En cualquier caso, es un factor común al TR de injertos obtenidos tanto por vía abierta como laparoscópica.

La evaluación del sindrome de isquemia-reperfusión se realizó mediante la determinación del FSR al desclampaje tras finalizar las anastomosis vasculares.

En ambos grupos el FSR postdesclampaje era inferior al FSR prenefrectomía. El análisis comparativo del FSR postTR en ambos grupos objetivó una disminución significativa del FSR medio durante la $1^{a}$ hora en el grupo LAP $(117,0 \mathrm{ml} / \mathrm{m})$ frente al grupo abierto $(159,7 \mathrm{ml} / \mathrm{m})$. Esta disminución era más acusada a los 5 minutos de inicio de la reperfusión, para continuar con una recuperación progresiva y prácticamente paralela entre ambos grupos durante la $1^{a}$ hora. El valor de FSR final fue significativamente inferior en el grupo LAP (Fig. 2).

De modo sintético, en el síndrome de isquemia-reperfusión se distinguen 2 periodos:

- El periodo de isquemia, en el que la falta de oxígeno a nivel mitocondrial ocasiona acidosis intracelular, y se produce una alteración de la membrana plasmática que conduce al edema celular y la muerte por citolisis ${ }^{63,64}$.

- El periodo de reperfusión, durante el cual se produce la llegada de oxígeno de forma importante a las células. Se originan los radicales libres de oxígeno ${ }^{65}$, que conducen a un aumento de la permeabilidad capilar, produciendo una disfunción del endotelio vascular, y disminución en la liberación de NO, que junto con factores quimiotácticos promueven un reclutamiento de polimorfonucleares al lugar reperfundido. La respuesta inflamatoria aguda iniciada se caracteriza por una cascada de citocinas proinflamatorias ${ }^{37}$, expresión de moléculas de adhesión e infiltración celular. Otros datos de la respuesta inflamatoria son menos agudos e incluyen la infiltración local por linfocitos T y macrófagos, así como la modificación de la expresión de los antígenos HLA ${ }^{37,65}$

Estudios recientes muestran, que además del proceso inflamatorio, la muerte celular por apoptosis se incrementa durante el sindrome de isquemia-reperfusión ${ }^{66-68}$

Todas estos mecanismos conducen a una disminución del FSR postreperfusión y al deterioro de la función del injerto ${ }^{69}$.

En nuestro estudio, el tiempo de isquemia fría fue idéntico en ambos grupos (24 h), mientras que el primer tiempo de isquemia caliente para el grupo abierto fue de 1,8 minutos frente a los 6,4 minutos del grupo LAP. Por tanto, el mayor tiempo de isquemia caliente sufrido por los injertos extraídos por laparoscopia, podría colaborar a la acentuación del sindrome de isquemia-reperfusión postTR. El menor tiempo de isquemia fría reduce la lesión de isquemia-reperfusión, lo que disminuye el daño del injerto mediado por citoquinas, asociadas a un proceso inflamatorio inespecífico. A su vez, la reducción de la lesión por isquemia-reperfusión, disminuye la susceptibilidad a respuestas aloinmunes específicas, que activando células T mediarian en el rechazo. Esto explicaria, que los injertos procedentes de donante vivo no relacionado con tiempo corto de isquemia fría, tengan mejor supervivencia que los de cadáver a igualdad de entidades ${ }^{20}$.

Este factor es trascendente, porque de hecho en la literatura está claramente documentado que la función inicial del injerto es tanto mejor cuanto mayor es el FSR pos-revascularización. Esto es, a menor intensidad del sindrome de isquemia-reperfusión mejor función renal ${ }^{70,71}$.

Asimismo, la severidad de este sindrome condiciona la incidencia de NTA, habiendo sido incluso sugerida una mayor frecuencia de aparición de rechazo agudo y crónico, aunque este extremo está sometido a controversia, y menor supervivencia del injerto tras 1 año de seguimiento ${ }^{64}$. 
La disminución del FSR postTR como consecuencia del daño por isquemia-reperfusión es un fenómeno referido ampliamente en la literatu$\mathrm{ra}^{72,73}$ Brentan et al. $^{74}$, en su modelo animal demuestran una disminución del FSR postTR con una lenta recuperación que tras 1 hora se mantiene por debajo del nivel normal, guardando los niveles de FSR correlación con la función y la diuresis durante la semana posterior al TR.

Es importante conocer si el neumoperitoneo condiciona o modula la lesión por isquemia reperfusión y la función inicial del injerto. Sasaki et al. ${ }^{33}$ estudiaron el efecto de la isquemia caliente en la función inicial del injerto en 100 casos de NLDV, demostrando, que tiempos superiores a 10 minutos se asociaban con valores significativamente superiores de $\mathrm{Cr}$ sérica a la semana del trasplante. Comparado con la cirugía abierta, los niveles de $\mathrm{Cr}$ se han visto elevados los primeros 3-6 días postrasplante ${ }^{32}$; que parecía asociarse a una mayor tiempo de isquemia caliente y a la curva de aprendizaje. No obstante, a los 3, 6 y 12 meses no existía diferencia significativa entre los dos grupos. Un estudio reciente no demuestra diferencias significativas en la función inicial y final del injerto con tiempos de isquemia calientes inferiores a 5 minutos, entre 5 y 10 minutos y superiores a 10-15 minutos $^{75}$.

El análisis comparativo de la evolución funcional del injerto, demostró que existian diferencias significativas durante la primera semana postTR entre el grupo LAP y Abierto. Los valores de $\mathrm{Cr}$ se determinaron de forma basal, y a los dias 1, 3, 5 y 7 del trasplante, y fueron significativamente superiores en el grupo LAP frente a los del grupo Abierto. Los niveles de $\mathrm{Cr}$ en el grupo LAP se mantuvieron elevados durante los 3 primeros dias, para posteriormente normalizarse aunque alcanzando cifras de $\mathrm{Cr}$ superiores a las del grupo Abierto (Fig. 3).

Habria varias diferencias en la técnica laparoscópica frente a la cirugía abierta, potencialmente involucradas en la disfunción inicial de los injertos $^{32,57,68}$ : la lesión mecánica del riñón durante la nefrectomía, el tiempo de la primera isquemia caliente y la isquemia renal "relativa" ocasionada por la disminución del FSR ${ }^{76,77}$.

- La disección laparoscópica parece aumentar la manipulación intraoperatoria del órgano, lo que podría ocasionar el espasmo de la arteria renal, y durante las maniobras de extracción puede sufrir traumatismo mecánico ${ }^{32}$.

- El primer tiempo de isquemia caliente en el grupo LAP fue inferior a $7 \mathrm{~min}$. Tiempos de isquemia caliente inferiores a $10 \mathrm{~min}$ no se ha demostrado que produzcan un retraso en la función inmediata del injerto ${ }^{33,75}$. En la situación habitual del TR con donante vivo, el tiempo de isquemia fría es muy corto. Sin embargo, en el presente modelo experimental ha sido de $24 \mathrm{~h}$, lo que se aparta de la práctica clínica convencional y podría tener un efecto aditivo a la isquemia renal relativa inducida por el neumoperitoneo, justificando los valores más elevados de Cr durante la primera semana en el grupo LAP.

- Valores de PIA durante el neumoperitoneo entre 12 y $16 \mathrm{mmHg}$ permiten una aceptable exposición anatómica y cambios hemodinámicos tolerables y reversibles en adultos. Sin embargo, en los pacientes pediátricos se recomienda limitar la PIA a 6-10 mmHg para evitar el desarrollo de un síndrome compartimental. La PIA de $15 \mathrm{mmHg}$ considerando el peso y el volumen abdominal, del animal son elevadas, si bien en la mayoría de trabajos experimentales con cerdos se suele emplear esa PIA. El sindrome compartimental inducido por una PIA de $15 \mathrm{mmHg}$ es más intenso en un animal de $20 \mathrm{~kg}$ de peso (situación potencialmente semejante a la infancia en la práctica clínica) que en un humano cuyo peso puede oscilar entre 60 y $80 \mathrm{~kg}$. Por otro lado, la configuración anatómica del cerdo con una celda de Gerota sin contenido graso, posiblemente atenúa menos la compresión del parénquima renal por la hiperpresión intraabdominal que en el humano con importante cantidad de grasa perirrenal. Finalmente, la expansión moderada de la volemia inducida en los animales de nuestro modelo experimental $(10 \mathrm{ml} / \mathrm{Kg} / \mathrm{h})$, puede haber sido insuficiente para compensar el efecto sobre el FSR ocasionado por una PIA elevada para el espacio retroperitoneal del cerdo ${ }^{76,77}$.

La experiencia inicial con NLDV apuntaba a un posible aumento de las incidencias de función retrasada del injerto con necesidad de diálisis y de las complicaciones ureterales después del TR. Sin embargo, una vez superada la curva de aprendizaje, la incidencia de tales complicaciones se reducen ${ }^{78,79}$. 
La influencia en la función inmediata del injerto de la nefrectomía laparoscópica frente a la cirugía abierta está sometida a controversia. Nogueira et al. ${ }^{32}$, comparando retrospectivamente la NLDV con la cirugía abierta, refieren niveles de creatinina más elevados los primeros 7 días postTR en los injertos extraídos por laparoscopia. No obstante, a los 3, 6 y 12 meses no existian diferencias significativas entre los dos grupos. Incluso, en algunas series tras el año del trasplante, se han descrito niveles inferiores de creatinina en el grupo de nefrectomía laparoscópica frente al de nefrectomía abierta ${ }^{27,32-35}$.

Ratner el al. ${ }^{55}$, también describen retrospectivamente un descenso paulatino de la creatinina durante los 3 primeros días tras el TR en los injertos extraídos por vía laparoscópica frente a la vía abierta. No obstante, al $4^{\circ}$ día no había diferencia significativa entre ambos.

Stifelman et al. ${ }^{45}$, cuando comparan retrospectivamente su experiencia con NLDV mano asistida y nefrectomía abierta, no encuentran diferencias entre el nadir de creatinina, el aclaramiento de creatinina y el tiempo transcurrido hasta el nadir de creatinina en ambos grupos.

Pero uno de los trabajos más importantes publicados en este sentido es el de Wolf et $\mathrm{al}^{43}$, por ser un estudio randomizado y prospectivo, en el cual se analizan los niveles de creatinina diarios durante la primera semana, al mes y a los tres meses. No encontraron diferencias en la función renal del injerto, bien sea el riñón obtenido mediante nefrectomía laparoscópica mano asistida o mediante cirugía abierta. No obstante, lo más importante es que ni al principio ni actualmente, se han encontrado diferencias en la función renal a largo término, analizado al año y a los tres años, dependiendo de la forma de extracción renal (laparoscópica o cirugía abierta) ${ }^{36}$.

\section{CONCLUSIONES}

Los riñones extraídos por vía laparoscópica sufren un deterioro significativo de la función durante la primera semana postrasplante, frente a aquellos riñones extraídos vía abierta. Los niveles de $\mathrm{Cr}$ en el grupo laparoscópico se mantuvieron elevados durante los 3 primeros dias, para posteriormente normalizarse, aunque alcanzando cifras de $\mathrm{Cr}$ superiores a las del grupo abierto.
Los injertos renales extraídos vía laparoscópica presentan una acentuación del síndrome de isquemia-reperfusión manifestado por un FSR postdesclampaje inferior al FSR prenefrectomía y al de los riñones extraídos vía abierta. Esta disminución era más acusada a los 5 minutos de inicio de la reperfusión, para continuar con una recuperación progresiva y prácticamente paralela entre ambos grupos durante la $1^{a}$ hora. El valor de FSR final fue significativamente inferior en el grupo laparoscópico.

\section{REFERENCIAS}

1. UNOS: Facts about transplantation in the United States. www.unos.net.Accessed 2003.

2. Ojo AO, Hanson JA, Meier-Kriesche H, Okechukwu CN, Wolfe RA, Leichtman AB, et al. Survival in recipients of marginal cadaveric donor kidneys compared with other recipients and wait-listed transplant candidates. J Am Soc Nephrol. 2001;12(3):589-597.

3. Wolfe RA, Ashby VB, Milford EL, Ojo AO, Ettenger RE, Agodoa LY, et al. Comparison of mortality in all patients on dialysis, patients on dialysis awaiting transplantation, and recipients of a first cadaveric transplant. N Engl J Med. 1999;341(23): 1725-1730.

4. Ortega AE, Peters JH, Incarbone R, Estrada L, Ehsan A, Kwan Y, et al. A prospective randomized comparison of the metabolic and stress hormonal responses of laparoscopic and open cholecystectomy. J Am Coll Surg. 1996; 183(3): 249-256.

5. Meier-Krische HU, Kaplan B. Waiting time on dialysis as the strongest modifiable risk factor for renal trasplant outcomes: a paired donor kidney analysis. Transplantation. 2002; 27: 1377-81.

6. World Almanac Education Group: Vital statistics. In: The World Almanac and Book of Facts 2003. Edited by W. A. McGeneran, L.P. Wiesenfeld and K. Seabrook. New York: World Almanac Books, pp 76 and 86, 2003.

7. UNOS: Facts about transplantation in the United States. www.unos.net.Accessed 2003.

8. Groth CG. Presidential address 2002: Organ transplantation as a patient service worldwide. Transplantation. 2003; 75(8):1098-1100.

9. Memoria de la ONT sobre la actividad de donación y trasplante de órganos en España. 2006.

10. Wolfe RA, Ashby VB, Milford EL, Ojo AO, Ettenger RE, Agodoa LY, et al. Comparison of mortality in all patients on dialysis, patients on dialysis awaiting transplantation, and recipients of a first cadaveric transplant. N Engl J Med. 1999;341(23):1725-1730.

11. Haberal M, Karakayali H, Moray G, Demirag A, Yildirim S, Bilgin N. Long-term follow-up of 102 living kidney donors. Clin Nephrol. 1998;50(4):232-235.

12. Westlie L, Fauchald P, Talseth T, Jakobsen A, Flatmark A. Quality of life in Norwegian kidney donors. Nephrol Dial Transplant. 1993;8(10):1146-1150.

13. Merlin TL, Scott DF, Rao MM, Wall DR, Francis DM, Bridgewater FH, et al. The safety and efficacy of laparoscopic live donor nephrectomy; a systematic review. Transplantation. 2000;70(12):1659-1666. 
14. Foss A, Leivestad T, Brekke IB, Fauchald P, Bentdal O, Lien B, et al. Unrelated living donors in 141 kidney transplantations. Transplantation. 1998;66(1):49-52.

15. D’Alessandro AM, Pirsch JD, Knechtle SJ, Odorico JS, Van der Werf WJ, Collins BH, et al. Living unrelated renal donation: the University of Wisconsin experience. Surgery. 1998;124(4):604-611 discussion 610-611.

16. United Network for Organ Sharing (UNOS) and the Division of Transplantation, Bureau of Health Resources and Services Administration: Annual Report of the U.S. Scientific Registry of Transplant Recipients and Organ Procurement and Transplantation Network-Transplant Data. Rockville, Md, U.S. Department of Health and Human Services, 1998.

17. Waples MJ, Belzer FO, Uehling DT. Living donor nephrectomy: a 20-year experience. Urology. 1995;45(2):207-210.

18. Mullins CD, Thomas SK, Pradel FG, Bartlett ST. The economic impact of laparoscopic living-donor nephrectomy on kidney transplantation. Transplantation. 2003;75(9):1505-1512.

19. DeMarco T, Amin M, Harty JI. Living donor nephrectomy: Factors influencing morbidity. J Urol. 1982;127(6):10821085.

20. Ojo AO, Hanson JA, Meier-Kriesche H, Okechukwu CN, Wolfe RA, Leichtman AB, et al. Survival in recipients of marginal cadaveric donor kidneys compared with other recipients and wait-listed transplant candidates. J Am Soc Nephrol. 2001;12(3):589-597.

21. Hamilton BD, Chow GK, Stowe NT: The effect of renal vein compression on renal function: A canine model for laparoscopic surgery. J Endourol. 1997;11: s53.

22. Schuessler WW, Vancaillie TG, Reich H, Griffith DP. Transperitoneal endosurgical lymphadenectomy in patients with localized prostate cancer. J Urol. 1991;145 (5):988-991.

23. Ohishi A, Suzuki H, Nakamoto H, Katsumata H, Hayashi $\mathrm{K}$, Ryuzaki M, et al. Status of patients who underwent uninephrectomy in adulthood more than 20 years ago. Am J Kid Dis. 1995;26(6):889-897.

24. Sánchez-de-Badajoz E, Díaz-Ramírez F, Vara-Thorbeck C: Endoscopic varicocelectomy. J Endourol. 1990;4:371-374.

25. Alcaraz A, Rosales A, Guirado L, Díaz J, Musquera M, Villavicencio H. Early experience of a living donor kidney transplant program. Eur Urol. 2006; 50(3):542-547; discussion 547-548. Epub 2006.

26. Bay WH, Hebert LA. The living donor in kidney transplantation. Ann Intern Med. 1987;106(5):719-727.

27. Najarian JS, Chavers BM, McHugh LE, Matas AJ. 20 years or more of follow-up of living kidney donors. Lancet. 1992;340(8823):807-810.

28. Jones KW, Peters TG, Walker GW. Anterior-retroperitoneal living donor nephrectomy: technique and outcomes. Am Surgeon. 1999;65(3):197-204.

29. Matas AJ, Bartlett ST, Leichtman AB, Delmonico FL. Morbidity and mortality after living kidney donation, 19992001: survey of United States transplant centers. Am J of Transplant. 2003;3(7):830-834.

30. Ratner LE, Hiller J, Sroka M, Weber R, Sikorsky I, Montgomery RA. Laparoscopic live donor nephrectomy removes disincentives to live donation. Transplant Proc. 1997;29 (8):3402-3403.

31. Daemen JW, Oomen AP, Janssen MA, van de Schoot L, van Kreel BK, Heineman E, et al. Glutathione S-transferase as predictor of functional outcome in transplantation of machine-preserved non-heart-beating donor kidneys. Transplantation. 1997;63(1):89-93.
32. Roodnat JI, van Riemsdijk IC, Mulder PG, Doxiadis I, Claas FH, IJzermans $\mathrm{JN}$, et al. The superior results of living- donor renal transplantation are not completely caused by selection or short cold ischemia time: a single-center, multivariate analysis. Transplantation. 2003;75(12): 2014-2018.

33. Khauli RB, Hussein M, Shaar A, Madi R, Medawar W, Habbal A, et al. A prospective evaluation of laparoscopic donor nephrectomy versus open donor nephrectomy. Transplant Proc. 2003;35(7):2552.

34. Sasaki TM, Finelli F, Bugarin E, Fowlkes D, Trollinger J, Barhyte DY, et al. Is laparoscopic donor nephrectomy the new criterion standard?. Arch Surg. 2000;135(8):943-947.

35. London E, Rudich S, McVicar J, Wolfe B, Perez R. Equivalent renal allograft function with laparoscopic versus open live donor nephrectomies. Transplant Proc. 1999;31(1-2):258-260.

36. Philosophe B, Kuo PC, Schweitzer EJ, Farney AC, Lim JW, Johnson LB, et al. Laparoscopic versus open donor nephrectomy. Transplantation. 1999;68(4):497-502.

37. Gill IS, Clayman RV, McDougall E: Advances in urological laparoscopy. J Urol. 1995;154(4):1274-1294.

38. Clayman RV, McDougall EM, Kerbl K. Laparoscopic nephrectomy: transperitoneal vs retroperitoneal. J Urol. 1994; 151:342.

39. Wadström J, Lindström P. Hand-assisted retroperitoneoscopic living-donor nephrectomy: initial 10 cases. Transplantation. 2002;73(11): 1839-1840.

40. Yang SC, Rha KH, Kim YS, Kim SI, Park K. Retroperitoneoscopy-assisted living donor nephrectomy: 109 cases. Transplant Proc. 2001;33(1-2):1104-1105.

41. Gaston KE, Moore DT, Pruthi RS. Hand assisted laparoscopic nephrectomy: prospective evaluation of the learning curve. J Urol. 2004;171(1):63-67.

42. Wolf JS Jr, Tchetgen MB, Merion RM: Hand-assisted laparoscopic live donor nephrectomy. Urology. 1998;52(5):885887.

43. Wolf J, Merion R, Leichtman A: Randomized controlled trial of hand-assisted laparoscopic versus open surgical live donor nephrectomy. Transplantation. 2001;72(2):284290.

44. Suzuki K, Ushiyama T, Ishikawa A, Mugiya S, Fujita K. Retroperitoneoscopy assisted live donor nephrectomy: the initial 2 cases. J Urol. 1997;158(4):1353-1356.

45. El-Galley R, Hood N, Young CJ, Deierhoi M, Urban DA. Donor nephrectomy: a comparison of techniques and results of open, hand assisted and full laparoscopic nephrectomy. J Urol. 2004;171(1):40-43.

46. Jacobs SC, Cho E, Foster C, Liao P, Bartlett ST. Laparoscopic live donor nephrectomy: the University of Maryland 6-year experience. J Urol. 2004;171(1):47-51.

47. Nogueira JM, Cangro CB, Fink JC, Schweitzer E, Wiland A, Klassen DK, et al. A comparison of recipient renal outcomes with laparoscopic versus open live donor nephrectomy. Transplantation. 1999;67(5):722-728.

48. Simforoosh N, Bassiri A, Ziaee SA, Tabibi A, Salim NS, Pourrezagholi F, et al. Laparoscopic versus open live donor nephrectomy: the first randomized clinical trial. Transplant Proc. 2003;35(7):2553-2554

49. Kavoussi LR. Laparoscopic donor nephrectomy. Kidney Int. 2000;57(5):2175-2186.

50. Gaur DD, Agarwal DK, Purohit KC. Retroperitoneal laparoscopic nephrectomy: initial case report. J Urol. 1993; 149(1):103-105. 
51. Doublet JD, Peraldi MN, Monsaint H, Tligui M, Sraer JD, Gattegno B, et al. Retroperitoneal laparoscopic nephrectomy of native kidneys in renal transplant recipients. Transplantation. 1997;64(1):89-91.

52. Hoznek A, Olsson LE, Salomon L, Saint F, Cicco A, Chopin $\mathrm{D}$, et al. Retroperitoneal laparoscopic living-donor nephrectomy. Eur Urol. 2001;40(6):614-618.

53. Guillonneau B, Ballanger P, Lugagne PM, Valla JS, Vallancien G. Laparoscopic versus lumboscopic nephrectomy. Eur Urol. 1996;29(3):288-291.

54. Burgos FJ, Pascual J, Briones G, Cuevas B, Villafruela J, Correa C, et al. Influence of laparoscopic live donor nephrectomy in ischemia-reperfusion syndrome and renal function after kidney transplantation: an experimental study. Transplant Proc. 2003;35(5):1664-5.

55. Ayala A, Ertel W, Chaudry IH. Trauma-induced suppression of antigen presentation and expression of major histocompatibility class II antigen complex in leukocytes. Shock. 1996;5(2):79-90.

56. Meldrum DR, Sheridan BC, Cleveland JC Jr, Fullerton DA, Banerjee A, Harken AH. Neutrophils are required for endotoxin-induced myocardial cross-tolerance to ischemiareperfusion injury. Arch Surg. 1996;131(11):1203-1208.

57. Harman PK, Kron IL, McLachlan HD, Freedlender AE, Nolan SP. Elevated intraabdominal pressure and renal function. Am Surg. 1982;196(5):594-597.

58. Kheirabadi BS, Tuthill D, Pearson R, Bayer V, Beall D, Drohan W, et al. Metabolic and hemodynamic effects of CO2 pneumoperitoneum in a controlled hemorrhage model. J Trauma. 2001;50(6):1031-1043.

59. Page $\mathrm{IH}$ : The production of persistent arterial hypertension by cellophane perinephritis. JAMA. 1939;113:2046-2048.

60. Vucasin A, Schichman S, Hom D: The mechanism of oliguria associated with intrabdominal insufflation. J Urol. 1995; $151: 34$

61. Yamada T, Murase N, Maeda T, Ye Q, Sakamoto T, Terakura M. Protective effect of TNF-alpha and IL-1 beta inhibitor FR167653 on ischemia-reperfusion injury in rat small intestinal transplantation. Transplant Proc. 1998; 30(6):2638

62. Linares A, Burgos FJ, Villafruela J, Zamora J, Pascual J, Marcén R, et al.: "Estudio comparativo de las modificaciones del flujo sanguíneo renal (FSR) inducidas por la nefrectomía laparoscópica y abierta. Modelo experimental". Endosurgery; 3, 2005.

63. Weight SC, Bell PR, Nicholson ML: Renal ischemia-reperfusion injury. Br J Surg 1996; 83: 162-170.

64. Matsuyama M, Yoshimura R, Akioka K, Okamoto M, Ushigome H, Kadotani Y, et al. Tissue factor antisense oligonucleotides prevent renal ischemia-reperfusion injury. Transplantation. 2003;76(5):786-791.

65. Castaneda MP, Swiatecka-Urban A, Mitsnefes MM, Feuerstein D, Kaskel FJ, Tellis V, et al. Activation of mitochondrial apoptotic pathways in human renal allografts after ischemia-reperfusion injury. Transplantation. 2003;76(1):50-54.

66. Johannsen G, Andersen M, Juhl B. The effect of general anaesthesia on the hemodynamic events during laparoscopy with CO2 insuflation. Acta Anaes Scand. 1989;33(2): 132-136.
67. Ekman LG, Abrahamsson J, Biber B, Forssman L, Milsom I, Sjöqvist BA. Hemodynamic changes during laparoscopy with positive end-expiratory pressure ventilation. Acta Anaes Scand. 1988;32(6):447-453.

68. Iwase $\mathrm{K}$, Takenaka H, Yagura A, Ishizaka T, Ohata $\mathrm{T}$, Takagaki M, et al. Hemodynamic changes during laparoscopic cholecystectomy in patients with heart disease. Endoscopy. 1992;24(9):771-773.

69. Salvemini D, Seibert K, Masferrer JL, Misko TP, Currie MG, Needleman P. Endogenous nitric oxide enhances prostaglandin production in a model of renal inflammation. J Clin Invest. 1994;93(5):1940-1947.

70. Matsuno N, Kozaki K, Degawa H, Narumi Y, Suzuki N, Kikuchi K, et al. Importance of machine perfusion in kidney preservation. Tansplant Proc. 1999;31(5):2004-2005.

71. Matsuno N, Sakurai E, Uchiyama M, Kozaki K, Miyamoto $\mathrm{K}$, Kozaki M. Usefulness of machine perfusion preservation for non heart-beating donors in kidney transplantation. Transplant Proc. 1996;28(3):1551-1552.

72. Ogawa T, Nussler AK, Tuzuner E, Neuhaus P, Kaminishi M, Mimura Y, et al. Contribution of nitric oxide to the protective effects of ischemic preconditioning in ischemiareperfused rat kidneys. J Lab Clin Med. 2001;138(1):5058.

73. Jamart J, Lambotte L. Differential effects of swelling and anoxia on kidney function and its consequences on the mechanism of action of intracellular organ preservation solutions. Transplantation. 1982;34(4):176-182.

74. Bretan PN Jr, Lobo E, Chang JA, Dumitrescu O, Miller B, Yen TS. Assessment of preservation induced reperfusion injury via intraoperative renal transplant blood flow and endothelin concentration studies. J Urol. 1997;158(3 Pt 1):714-718.

75. Soulsby RE, Evans LJ, Rigg KM, Shehata M. Warm ischemic time during laparoscopic live donor nephrectomy: effects on graft function. Transplant Proc. 2005;37(2):620622.

76. Burgos FJ, Linares A, Pascual J, Marcen R, Villafruela J, Zamora J, et al. Modifications of renal blood flow and srum interleukin levels induced by laparoscopic and open living donor nephrectomies for kidney transplant. An experimental study in pigs. Transplant.Proc. 2005;37(9):3676-3678.

77. Linares A: Estudio comparativo de la función del trasplante renal con donante vivo mediante nefrectomía convencional y laparoscópica. Influencia en el síndrome de isquemiareperfusión. Modelo experimental. Tesis doctoral. Madrid: Universidad de Alcalá. Facultad de Medicina. 2004.

78. Novick AC. Laparoscopic live donor nephrectomy. Urology. 1999;53(4):668-670.

79. Abreu SC, Goldfarb DA, Derweesh I, Thornton J, Urbain JL, Mascha E, et al. Factors related to delayed graft function after laparoscopic live donor nephrectomy. J Urol. 2004;171(1):52-57.

Correspondencia autora: Dra. A.I. Linares Quevedo

Servicio de Urología. Hospital de Fuenlabrada

Camino del Molino, s/n - 28942 Fuenlabrada (Madrid)

Tel.: 916006000

E-mail autora: analinaresquevedo@hotmail.com

Información artículo: Original 